\title{
A BIOINSPIRED HYBRID SILICA-PROTEIN MATERIAL WITH ANTIMICROBIAL ACTIVITY BY IRON UPTAKE
}

Fernando Carmona, Daniela Mendoza, Alicia Megía Fernández ,Francisco Santoyo-Gonzalez and José M. Domínguez-Vera

\begin{abstract}
A silica-protein hybrid material has been prepared by simultaneous covalent deposition of apoferritin and lactoferrin on functionalized silica. This material exhibits strong antibacterial activity against E. coli due to its high ironuptake capacity.
\end{abstract}

As bacteria are able to infect almost all tissues in the human body, they cause a spectrum of illnesses ranging from common skin infections to severe and fatal diseases. Indeed, bacterial infection is one of the leading causes of death in the world. Furthermore, some bacteria have developed aremarkable ability to resist currently available antimicrobials, thus highlighting the need for the development of new antimicrobial agents. A promising strategy in this respect involves inhibiting their ability to obtain the nutrients required for growth. ${ }^{1}$ An effective route to combat bacterial infection is to create materials that can compete with bacteria for iron, thereby depriving them of this metal, which they need to proliferate. However, such a route must be more efficient in terms of iron uptake than siderophores, low-molecular-weight iron chelators that are produced and exported by bacteria during periods of nutrient deprivation. ${ }^{2}$ A good alternative to the creation of new materials for this purpose is to mimic existing and efficient biological systems. Animals closely regulate iron levels ${ }^{3}$ and sequester this nutrient as a mechanism of preventing bacterial proliferation. Two kinds of proteins, namely transferrins (including lactoferrin), which are glycoproteins that remove extracellular Fe(III) due to their high Fe(III) affinity, ${ }^{4,5}$ and ferritin, which stores the iron that is not required for immediate metabolic purposes, play a crucial role in this mechanism.,

Lactoferrin is considered to be one of the key components of the immune system, partly due to its high affinity for Fe(III), ${ }^{8,9}$ and ferritin consists of a spherical protein shell (apoferritin) surrounding an aqueous cavity that incorporates Fe(II), which is oxidized during its journey to the cavity to form a Fe(III) mineral core 
therein. ${ }^{6,7}$ The apoferritin shell is assembled from 24 polypeptide chains of two types, namely heavy (H) (21 kDa) and light (L) subunits (19 kDa). It has traditionally been thought that H-subunits play a key role in $\mathrm{Fe}(\mathrm{II})$ oxidation as they contain catalytic ferroxidase centres, whereas L-subunits are associated with Fe(III) nucleation. ${ }^{6}$ However, it is interesting to note that pure L ferritin has not been found in iron storage organs. All known ferritins always contain at least a small amount of $\mathrm{H}$ subunits (10\% in the liver, for example). As such, it seems that the iron uptake and storage process requires the oxidative activity of at least a small number of $\mathrm{H}$ subunits. Nevertheless, pure or H-rich ferritin is frequently found in humans, especially in breast milk, the heart or serum, probably due to its strong ferroxidase activity, which helps to protect against toxic Fe(II). ${ }^{10}$

In light of these findings, we considered that $\mathrm{H}$-apoferritin and lactoferrin could be an effective combination for iron uptake. Interestingly, breast milk contains both lactoferrin and apoferritin, the latter of which is an H-rich or H-pure apoferritin. ${ }^{10}$ As a result, we supposed that a material capable of confining both $\mathrm{H}$-apoferritin and lactoferrin could constitute an effective system for Fe(II) uptake as Fe(II) would be oxidized by apoferritin to Fe(III), some of which would be stored in its cavity, with the remainder, and any available Fe(III), being captured by lactoferrin. In this sense, it is interesting to note that most previous iron uptake strategies based on molecular recognition technologies ${ }^{11}$ involve solids containing immobilized molecules with a well-known affinity for Fe(III), such as desferrioxamine, ${ }^{12} 3$-hydro-xypyridin-4-ones, ${ }^{13}$ or EDTA derivatives. ${ }^{14}$ Despite the fact that free Fe(II) is markedly more dangerous than Fe(III) as it catalyses the production of reactive oxygen species, which are extremely powerful oxidizing agents that are capable of causing extensive cell damage, no hybrid solids with iron(II) molecules have been reported.

The aim of this work was therefore to create new materials containing both apoferritin (especially $\mathrm{H}$ pure) and lactoferrin to remove iron from the media, thus depriving pathological microorganisms of this metal. To achieve this goal, we decided to use a vinyl sulfone silica functionalized with both proteins in a step-bystep manner. The resulting hybrid silica-protein material spatially confines both proteins and mimics breast milk to some extent, thus making it potentially useful as an antimicrobial agent due to the cooperative effect of the apoferritin- 
lactoferrin combination for iron uptake.

The functionalization of silica with vinyl sulfone commences with the silanization of commercial silica followed by the Michael-type addition of a bis-vinyl sulfone. This process gives rise to a functionalized silica containing available vinyl sulfone groups to which different biomolecules can be covalently attached (ESI 1). ${ }^{15}$

We initially analysed the reactivity of each protein with the vinyl sulfone silica. Under similar conditions, the two apoferritins studied [horse spleen (HSFt) and Hrecombinant ( $\mathrm{HFt}$ )] showed a higher reactivity than lactoferrin, therefore we first carried out the immobilization of a default apoferritin followed, in a second step, by an excess of lactoferrin (Scheme 1). Both protein immobilizations were performed by incubating the aforementioned proteins with the vinyl sulfone silica in phosphate buffer at $\mathrm{pH}$ 7.5. After $24 \mathrm{~h}$ of incubation with apoferritin, the resulting solid was exhaustively washed with buffer $(1 \mathrm{M} \mathrm{NaCl})$ to remove the noncovalently immobilized apoferritin. UV-visible spectroscopic analysis of the wash solution showed practically no signal for both HSFt and HFt, thus confirming that almost all the apoferritin had bound covalently to the vinyl sulfone and that negligible amounts of protein were retained by non-covalent interactions (Fig. 1). Once the silica-apoferritin material had been isolated, it was incubated with an excess of lactoferrin and, after $24 \mathrm{~h}$, the solid containing both proteins (silica-Ft + Lf hereafter) was rinsed and dried at room temperature.

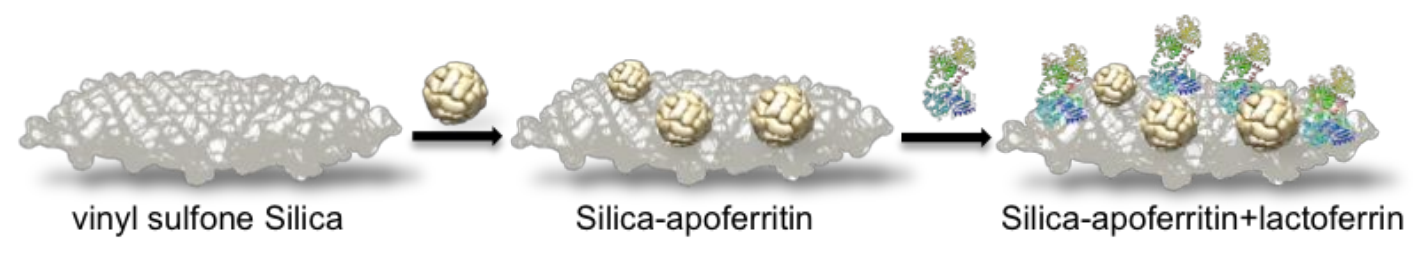

scheme 1 

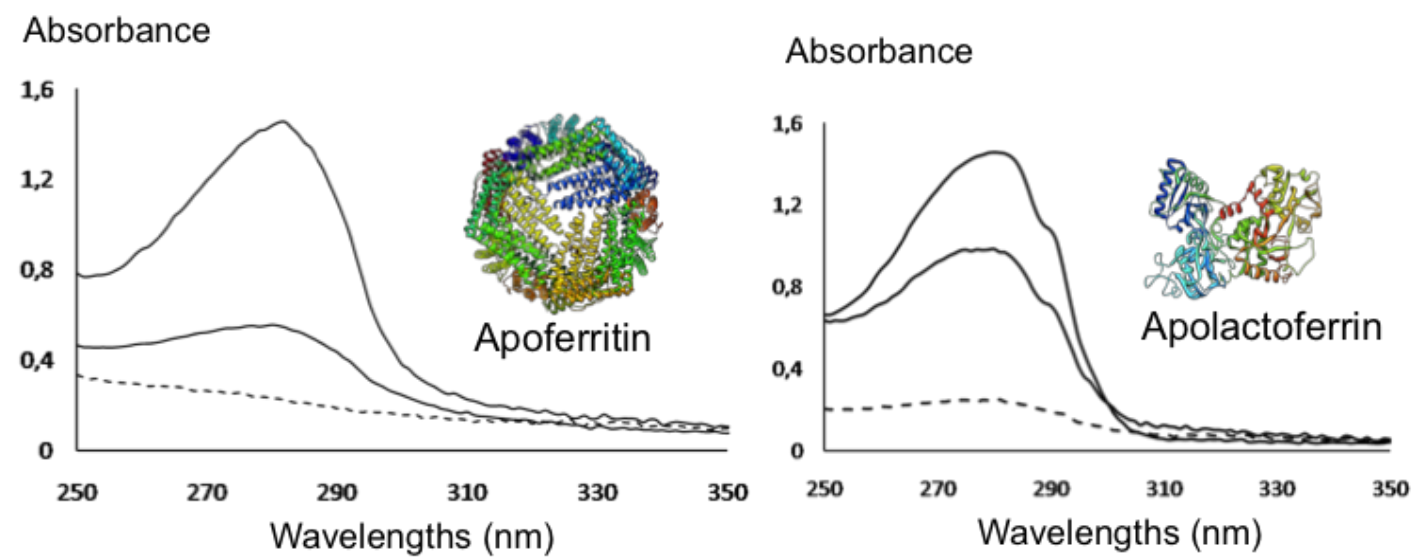

Fig 1. UV-visible spectra of proteins (H-apoferritin and lactoferrin) before and after incubation with silica (left) and silica-apoferritin (right). Dashed lines correspond to the spectra of the wash solution (non-covalent bound protein).

From the difference in the absorbance values of both protein solutions at $280 \mathrm{~nm}$ before and after incubation with vinyl sulfone silica, and after subtracting the small amount of protein removed upon washing the solid (Fig. 1), values of $33.0 \mathrm{mg}$ (for apoferritin) and $4.6 \mathrm{mg}$ per gram of vinyl sulfone silica (for lactoferrin) were obtained. Note that the absorbance at $280 \mathrm{~nm}$ for the solution obtained after washing the silica-HFt + Lf material is minimal. Furthermore, if this solution is mixed with the supernatant solution and dialyzed using a molecular weight cut-off of $100 \mathrm{kDa}$ (larger than the molecular weight of lactoferrin), no absorbance at 280 $\mathrm{nm}$ is observed at all, thus confirming that apoferritin is not displaced by the covalent attachment of lactoferrin and that both proteins are definitely fixed to the silica.

Once isolated, the hybrid silica-Ft + Lf materials were incubated with an Fe(II) solution ( $5 \mathrm{~mL}, 10 \mathrm{mM}$ ) and, after $6 \mathrm{~h}$, the resulting solids centrifuged and washed twice with buffer and $1 \mathrm{M} \mathrm{NaCl}$. The supernatant and wash solutions were mixed and the iron concentration measured by ICP to quantify the iron removal efficacy of the silica-Ft + Lf (silica-HSFt + Lf and silica-Ft + Lf). The results are shown in Table 1 along with values for control samples, functionalized vinyl sulfone silica and silica containing only one protein, either HSFt or Lf. 
The best values were obtained with recombinant $\mathrm{H}$-apoferritin and lactoferrin supported on the functionalized silica, which agrees with the expected existence of a cooperative effect of both proteins that leads to a very efficient iron uptake. This effect is lower in the silica-HSFt + Lf material as a result of the lower ferroxidase activity of horse spleen apoferritin with respect to H-pure apoferritin (silica-HFt + Lf). Likewise, it is interesting to highlight the low iron removal efficiency of the silica containing only lactoferrin (silica-Lf), which exhibits a similar yield to the silica containing no protein (silica). This is probably due to the high affinity of lactoferrin for Fe(III) but not for Fe(II). However, in the presence of a protein with ferroxidase activity, such as apoferritin, which oxidizes Fe(II) to Fe(III), lactoferrin multiplies its effectiveness and ultimately makes the apoferritin-lactoferrin combination the best option for iron uptake, especially if the apoferritin exhibits a high ferroxidase activity, as is the case for H-recombinant apoferritin.

In light of these promising results, we decided to check the antimicrobial activity of the silica-HFt + Lf material against E. coli. The antimicrobial activity of lactoferrin against $E$. coli has been reported both in solution ${ }^{16}$ or immobilized on the surface of a copolymer. ${ }^{17}$ However, the antimicrobial activity of our hybrid materials is based on the cooperative effect of two proteins: first by uptake and oxidation of Fe(II) (apoferritin) and ultimately the uptake of Fe(III) (apoferritin + lactoferrin). Our hybrid materials are therefore designed to act as antibacterial agents due to their ability to sequester Fe(II), the most toxic form of iron.

Table 1 Iron removed by the hybrid materials

\begin{tabular}{ll}
\hline Material & \%Fe removed \\
\hline Silica-HFt + Lf & $96 \pm 6$ \\
Silica-Ft + Lf & $76 \pm 7$ \\
Silica-HFt & $65 \pm 9$ \\
Silica-Lf & $15 \pm 5$ \\
Silica & $12 \pm 4$
\end{tabular}




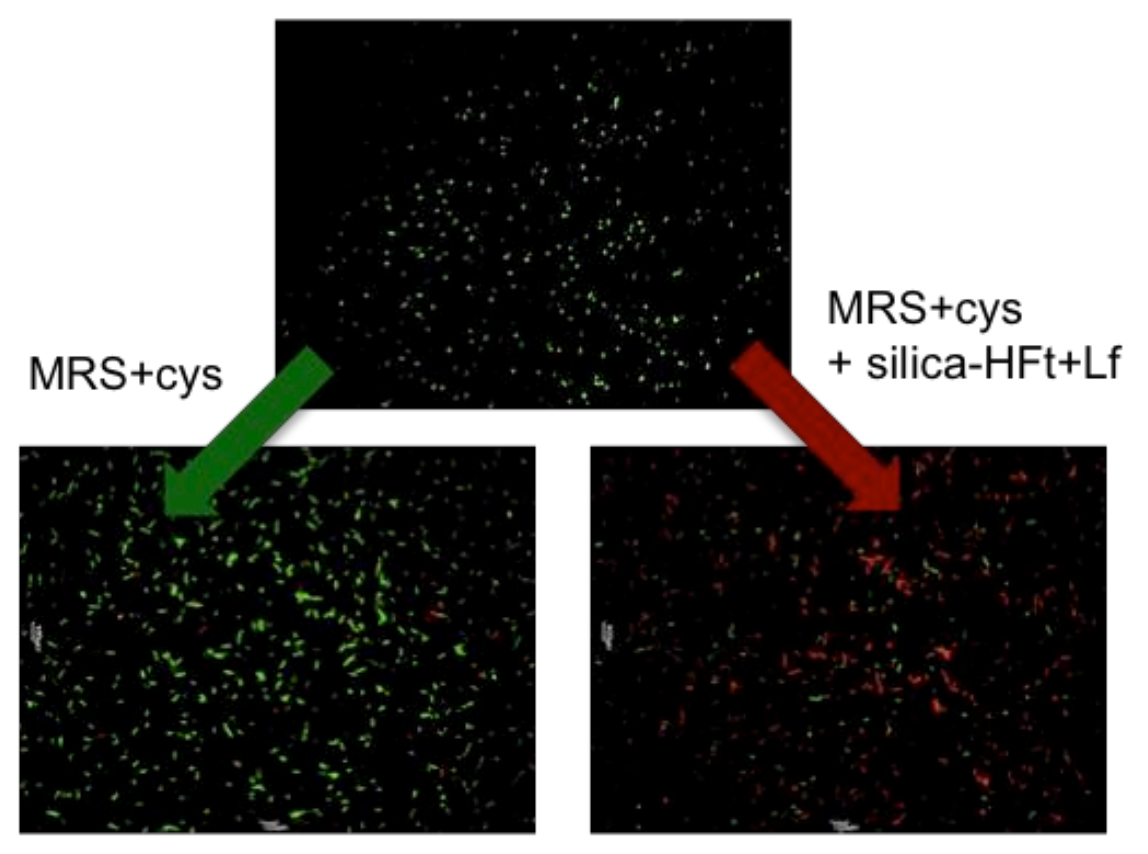

Fig 2. Fluorescence microscopy images of E. coli proliferation obtained using a Live/Dead Bacterial Viability Kit, where live and dead bacteria exhibit green and red emissions, respectively. Silica-HFt + Lf dramatically inhibits bacterial viability.

Bacterial inhibition by silica-HFt + Lf was quantified using the Live/Dead Bacterial Viability Kit, counting the number of live (green) and dead (red) bacteria by fluorescence microscopy. $\neq$ The average live/dead ratio (viability v) was used to quantify the bacterial inhibition effect of the hybrid silica-protein material in comparison with control experiments. As can be seen from Fig. 2, the silica-HFt + Lf material dramatically decreases the bacterial viability in MRS + cys (viability $\mathrm{v}=$ 0.9 ), a medium where these bacteria proliferate adequately (viability control $\mathrm{v}=$ 60.5).

Interestingly, the silica-Lf material, which only contains lactoferrin and removes small amounts of iron (Table 1), did not significantly inhibit bacterial viability ( $\mathrm{v}=$ 54.5). Likewise, in agreement with its higher iron uptake ability (Table 1), silicaHSFt inhibits more strongly than silica-Lf ( $\mathrm{v}=24.1)$ but is markedly less effective as an antibacterial agent than the silica material containing both proteins (silica$\mathrm{HFt}+\mathrm{Lf}$, viability $\mathrm{v}=0.9$ ). 
These results demonstrate the antibacterial activity of this novel hybrid silica-HFt + Lf material and confirm that the mechanism of antibacterial activity of this hybrid solid lies in the synergistic action of $\mathrm{H}$ apoferritin and lactoferrin for iron uptake.

\section{Conclusions}

The results of this study confirm our initial bio-inspired hypothesis that the confinement of apoferritin and lactoferrin supported on a material such as silica is a useful route for iron(II) uptake and therefore for use as an antimicrobial agent. A high antibacterial activity has been demonstrated against E. coli K-12, especially when using the silica containing both H-recombinant apoferritin and lactoferrin. Likewise, the correlation between bacterial inhibition and iron uptake confirms that the antimicrobial activity of these materials resides in their iron uptake capacity. Finally, we are convinced that this kind of material may find use as the active ingredient in creams or topical drugs for the treatment of microbial infections in cosmetics and personal hygiene.

This work was supported by MINECO and FEDER (project CTQ2009-09344), Junta de Andalucía (project P07-FQM-02899) and BIOSEARCH SA (contract PostBIO).

\section{Materials and methods}

\# The vinyl sulfone silica ${ }^{15}$ (ESI) (100 mg) was incubated with stirring with the protein $(2.5 \mathrm{~mL}, 0.01875 \mathrm{mM})$ in phosphate buffer at $\mathrm{pH} 7.5$ at $4{ }^{\circ} \mathrm{C}$. After $24 \mathrm{~h}$, the mixture was centrifuged at $10000 \mathrm{rpm}$ for $10 \mathrm{~min}$ at $20{ }^{\circ} \mathrm{C}$. The solid was washed with buffer containing $1 \mathrm{M} \mathrm{NaCl}$. Both supernatant solutions, from the first centrifugation and washed solid, were mixed and the protein concentration measured by the absorbance at $280 \mathrm{~nm}\left(\varepsilon^{280_{\mathrm{HS}}}=33900\right.$ and $\left.\varepsilon^{280_{\mathrm{HFt}}}=27900 \mathrm{M}^{-1} \mathrm{~cm}^{-1}\right)$ in the UV-visible spectrum. From the difference in the absorbance values at $280 \mathrm{~nm}$ in the initial protein solution and the mixture of supernatant solutions (after centrifugation and after washing), values of $33.0 \mathrm{mg}$ for apoferritin, and $4.6 \mathrm{mg}$ for apolactoferrin per gram of vinyl sulfone silica were obtained. 
Preparation of vinyl sulfone functionalized silica-lactoferrin + apoferritin (silica-HFt + Lf and silica-HSFt + Lf): the vinyl sulfone silica (100 mg) was incubated with stirring with apoferritin (HSFt or HFt) $(2.5 \mathrm{~mL}, 0.01 \mathrm{mM})$ in phosphate buffer at $\mathrm{pH}$ 7.5 at $4{ }^{\circ} \mathrm{C}$. After $24 \mathrm{~h}$, the mixture was centrifuged at $10000 \mathrm{rpm}$ for $10 \mathrm{~min}$ at $20{ }^{\circ} \mathrm{C}$. The solid was washed with buffer containing $1 \mathrm{M} \mathrm{NaCl}$. The resulting solid (silica-Ft or silica-HFt) was incubated with stirring with apolactoferrin $(2.5 \mathrm{~mL}, 0.05 \mathrm{mM})$ in phosphate buffer at pH 7.5 at $4{ }^{\circ} \mathrm{C}$. After 24 h, the mixture was centrifuged at 10000 rpm for 10 min at $20^{\circ} \mathrm{C}$. The solid was washed with buffer containing $1 \mathrm{M} \mathrm{NaCl}$ and dried at room temperature. Iron uptake by the hybrid silica-protein materials: the silica-protein (500 mg) was incubated with a solution of $\mathrm{Fe}\left(\mathrm{NH}_{4}\right)_{2}\left(\mathrm{SO}_{4}\right)_{2} 6 \mathrm{H}_{2} \mathrm{O}(5 \mathrm{~mL}$, $10 \mathrm{mM}$ ) for $6 \mathrm{~h}$ without stirring and then centrifuged at $10000 \mathrm{rpm}$ for $10 \mathrm{~min}$ at 20 ${ }^{o} \mathrm{C}$. The solid was washed with a $1 \mathrm{M} \mathrm{NaCl} \mathrm{solution.} \mathrm{The} \mathrm{supernatant} \mathrm{solution} \mathrm{from} \mathrm{the}$ centrifugation and the washed solution were mixed and the iron concentration measured by Inductive Coupled Plasma. From the ratio between final and initial iron concentration, the yield of iron removal was obtained for each sample (Table 1).

Inhibition of E. coli $K$-12: a bacteria culture (400 mL of $\left.1 \cdot 10^{7} \mathrm{CFU}\right)$ in MRS + cys was subjected to the hybrid material $(100 \mathrm{mg}$ ) for $16 \mathrm{~h}$. Quantification of bacterial proliferation was performed by using the Live/Dead Bacterial Viability Kits SYTO9 (green) and propidium iodide (red) (Invitrogen), counting the number of live (green) and dead (red) bacteria in a batch of three experiments using the Image-Pro Plus 6.0 software. The average live/dead ratio (viability $v$ ) was used to quantify the effect of the hybrid material on bacterial inhibition by comparing with control experiments where no silica-protein material was present. The presence of silica-HFt + Lf resulted in a decrease in viability to 0.9 with respect to control experiments where the viability was 60.5. Values of 24.1 and 54.5 were obtained for silica-HFt and silica-Lf, respectively. 


\section{Electronic Supplementary Information (ESI)}

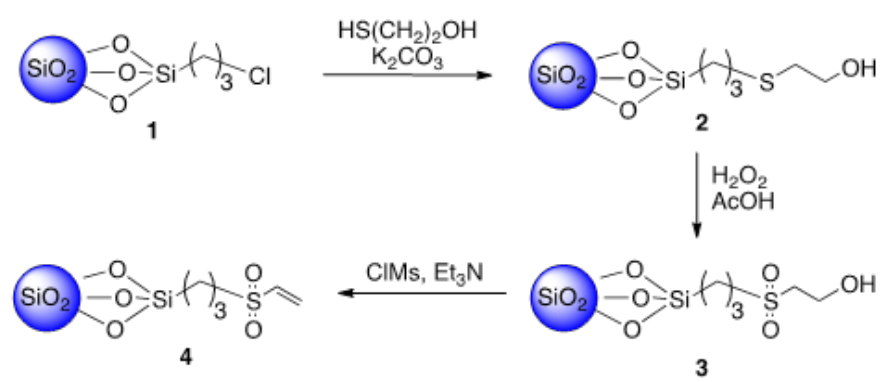

Scheme SI1. Synthetic route for the preparation of the vinyl sulfone silica.

Preparation of mercaptoethanol functionalized silica 2 (Scheme 1). Chloro functionalized silica 1 (5.0 g) was suspended in acetonitrile (40 mL) and then 2mercaptoethanol $(3.5 \mathrm{~mL})$ and potassium carbonate $(6.9 \mathrm{~g})$ were added. The magnetically stirred suspension was heated at $50{ }^{\circ} \mathrm{C}$ under an Ar atmosphere for $8 \mathrm{~h}$. The reaction mixture was filtered and the white powder washed with hot water $(2 \mathrm{x}$ $20 \mathrm{~mL})$ and finally with acetone $(2 \times 20 \mathrm{~mL})$ and dried under vacuum (1 $\mathrm{mmHg})$ at 50 ${ }^{o} C$ for 16 h giving the mercaptoethanol functionalized silica 2 (4.2 g).

Preparation of ethanol sulfone functionalized silica 3 (Scheme 1). Mercaptoethanol functionalized silica 2 (3.0 g) was suspended in acetic acid (6.0 $\mathrm{mL}$ ) and then hydrogen peroxide 33\% (15.0 mL) was added. The magnetically stirred reaction mixture was kept at room temperature for $24 \mathrm{~h}$ in the absence of light. After filtration, the white powder was washed with water $(2 \times 20 \mathrm{~mL})$, methanol $(2 \times 20$ $\mathrm{mL})$ and finally acetone $(2 \times 20 \mathrm{~mL})$, and dried under vacuum $(1 \mathrm{mmHg})$ at $50{ }^{\circ} \mathrm{C}$ for $16 \mathrm{~h}$ giving the ethanol sulfone functionalized silica $3(2.9 \mathrm{~g})$

Preparation of vinyl sulfone functionalized silica 4 (Scheme 1). Ethanol sulfone functionalized silica $3(2.5 \mathrm{~g}$ ) was suspended in anhydrous dichloromethane (40 $\mathrm{mL}$ ). The magnetically stirred suspension was cooled at $0{ }^{\circ} \mathrm{C}$ by means of an ice bath. Methanesulfonyl chloride $(0.9 \mathrm{~mL})$ and triethylamine $(3.5 \mathrm{ml})$ were then added. After the addition of the reagents, the reaction mixture was kept at room temperature for $7 \mathrm{~h}$ and then filtered. The white powder was washed with methanol $(2 \times 20 \mathrm{~mL})$ and acetone $(2 \times 20 \mathrm{~mL})$, and dried under vacuum (1mm $\mathrm{Hg})$ at $50{ }^{\circ} \mathrm{C}$ for $16 \mathrm{~h}$ giving the vinyl sulfone functionalized silica $4(2.4 \mathrm{~g})$. 
Proteins. Recombinant $H$ apoferritins were obtained from Molirom and was exhaustively dialyzed against milli-Q water using a Spectra/Por Float-A-Lyzer with a molecular weight cut-off (MWCO) of 300.000 Da. Horse spleen apoferritin and human apolactoferrin were purchased from Sigma and used as received.

\section{References}

1 J. J. Bullen, H. J. Rogers, P. B. Spalding and C. G. Ward, FEMS Immunol. Med. Microbiol., 2005, 43, 325-330.

2 E. D. Weinberg, Metallomics, 2010, 2, 732-740. 3 T. Zheng and E. M. Nolan, Metallomics, 2012, 4, 866-880.

4 H. J. Vogel, Biochem. Cell Biol., 2012, 90, 233-244.

5 E. N. Baker and H. M. Baker, Cell. Mol. Life Sci., 2005, 62, 2531-2539.

6 E. C. Theil, Curr. Opin. Chem. Biol., 2011, 15, 304-311; E. C. Theil, R. K. Beheraa and T. Takehiko, Coord. Chem. Rev., 2012, 257, 579-586.

7 N. E. Le Brun, A. Crow, M. E. P. Murphy, A. G. Mauk and G. R. Moore, Biochim. Biophys. Acta, 2010, 1800, 732-744; A. Lewin, G. R. Moore and N. E. Le Brun, Dalton Trans., 2005, 3597-3610.

8 R. D. Baynes and W. R. Bezwoda, Adv. Exp. Med. Biol., 1994, 357, 133-141.

9 D. Legrand, Biochem. Cell Biol., 2012, 90, 252-268.

10 P. Arosio, A. Ponzone, R. Ferrero, I. Renoldi and S. Levi, Clin. Chim. Acta, 1986, 161, 201-208.

11 H. Hiroshi, I. M. M. Rahman, K. Sanae, M. Teruya and F. Yoshiaki, Chemosphere, 2011, 82, 1161-1167.

12 N. A. A. Rossi, M. Ibrahim, J. K. Jackson, H. M. Burt, S. A. Horte, M. D. Scotta and J. N. Kizhakkedathua, Biomaterials, 2009, 30, 638-648.

13 J. Golenser, A. Domb, D. Teomim, A. Tsafack, O. Nisim, P. Ponka, W. Eling and Z. I. Cabantchik, J. Pharmacol. Exp. Ther., 1997, 281, 1127-1135.

14 F. Tian, E. A. Decker and J. M. Goddard, J. Agric. Food Chem., 2012, 60, 20462052. 
15 M. Ortega-MuÑoz, J. Morales-Sanfrutos, A. Megia-Fernandez, F. J. LopezJaramillo, F. Hernandez-Mateo and F. Santoyo-Gonzalez, J. Mater. Chem., 2010, 20, 7189-7196.

16 Y. Chih-Ching, C.-J. Shen, W.-H. Hsu, Y.-H. Chang, H.-T. Li, H.-L. Chen and C.-M. Chen, Biometals, 2011, 24, 585-594.

17 A. Oyane, Y. Yokoyama, M. Uchida and A. Ito, Biomaterials, 2006, 27, 32953303. 